\title{
Frictional and Biomechanical Forces and Biological Effects of a Trapezoidal Versus Rectangular Bracket
}

\author{
Teresa Cobo ${ }^{1}$, Alberto A. Suárez², Juan Cobo ${ }^{1}$ \\ ${ }^{1}$ Department of Surgery and Medical-Surgical Specialties, University of Oviedo and Instituto Asturiano de Odontología. \\ Asturias. España \\ ${ }^{2}$ Department of Mechanical Engineering. Escuela Politécnica Superior de Ingeniería. University of Oviedo. Asturias.
} España

\begin{abstract}
This paper presents a study of the frictional and biomechanical forces in a trapezoidal versus rectangular bracket. Moreover, a biological study based on the presence of periostin in the crevicular fluid was carried out. The main aim is to validate the novel and innovative bracket design (utility model U-2013 30854 applied for on: 8th July 2013. The scope of protection of this utility model covers Spain, France and Portugal). Results demonstrate that trapezoidal brackets transmit the forces to the tooth more uniformly and generate lower frictional forces, in an angle dependent manner until a maximum of $8^{\circ}$, than the rectangular ones.
\end{abstract}

Keywords: orthodontics, trapezoidal bracket, biomechanical, periostin.

(C) Copyright 2016 Authors - This is an Open Access article published under the Creative Commons Attribution License terms (http://creativecommons.org/licenses/by/3.0). Unrestricted use, distribution, and reproduction in any medium are permitted, provided the original work is properly cited.

\section{Introduction}

Orthodontic treatment is characterized by the application of forces to teeth with the intention of moving them to a predetermined desired position. To efficiently establish tooth movement, the forces applied to the teeth must be within a certain range. Forces which are too high can result in rapid, painful tooth movement or ankyloses, while forces are too low can result in slow or non-existent tooth movement.

Our hypothesis is the following: given that the archwire is not only supported by the bottom of the slot, but also by the walls, could a change in the morphology of the slot and/or archwire achieve an improvement in the behavioural relationship between the two in different scenarios? At first the bracket had a standard, rectangular face, and now, why not a trapezoidal face? (Fig. 1).

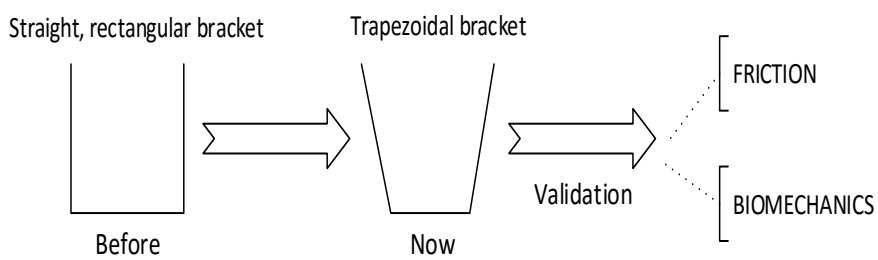

Figure 1. Evolution of the bracket.

\section{Method}

The strategy of the frictional and mechanical studies are summarized in the figure 2 .

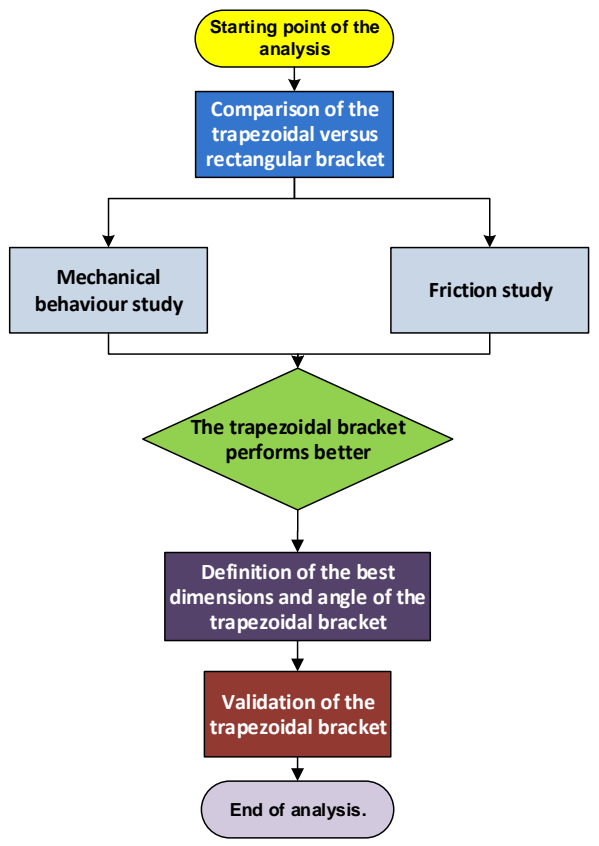

Figure 2. Stages of the frictional and mechanical studies. 


\subsection{Study of the mechanical behaviour of a trapezoidal versus rectangular bracket}

A numerical simulation was performed using the 3D finite elements method of three models of a dental bracket with different geometries for the cross-section of the archwire/slot: one "rectangular" in shape; one with trapezoidal geometry, $\alpha=5^{\circ}$ in the area of interest; and a third with trapezoidal geometry, $\alpha=8^{\circ}$ (Fig. 3).

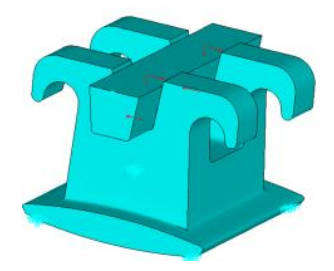

Figure 3. Trapezoidal bracket.

\subsection{Comparative study of arch wire friction on different bracket geometries. 1st case}

Three case studies were conducted of the bracket/archwire assembly, varying its geometry.

- Rectangular bracket with rectangular archwire.

- Trapezoidal bracket with rectangular archwire.

- Trapezoidal bracket with rectangular archwire supported on one side.

To obtain comparable results, all cases were simulated under the same conditions of friction, estimating a single coefficient of dynamic friction. Similarly, the forces applied to the archwire were always equal and constant for all studies, the estimated slip velocity between the bracket and archwire taking an equal and constant value in all cases.

\subsection{Comparative study of archwire friction on} different bracket geometries - varying the angle of the trapezoidal slot between $1^{\circ}$ and 10‥2nd case

Considering a common state of loading, a statics study of the archwire-bracket system was conducted on 4 possible hypotheses of impending movement.

Ten bracket geometries were studied in each of the four analysed cases, with the angle of the trapezoidal slot taking the values $1^{\circ}, 2^{\circ}, 3^{\circ}$... up to $10^{\circ}$.

After defining the relative position between archwire and bracket, different scenarios may be postulated based on hypotheses regarding the possible impending movement the archwire may suffer within the slot.

Specific reactions or frictional forces are generated for each case in each of these scenarios.
Four different cases were postulated, analysing in each one the 10 bracket geometries under study (trapezoidal slot angle varying between $1^{\circ}$ and $10^{\circ}$ ). These cases are described below.

1. The archwire is supported on one wall of the bracket slot, considering no state of impending movement. Hence, no frictional forces appear along the direction of the contact faces.

2. The archwire is supported on one wall of the bracket slot, considering a state of impending movement to exist on this surface. Hence, a frictional force appears along the direction of the slot wall.

3. The archwire is supported on the wall of the bracket slot, considering a state of impending movement to exist on the base of the slot (the archwire tends to be displaced in an anticlockwise direction. In this case, a frictional force appears along the direction of the base.

The archwire is supported on the wall of the bracket slot, considering a state of impending movement to exist simultaneously on the base and the slot wall (the archwire tends to turn in a clockwise direction. In this case, frictional forces appear along the direction of both the base and the contact slot wall.

\section{Results}

3.1. Results of the study of the mechanical behaviour of a trapezoidal versus rectangular bracket (table 1 and fig. 4)

Table 1. Results of the mechanical study.

\begin{tabular}{|c|c|c|}
\hline & $\begin{array}{c}\text { RESULTS } \\
\text { Max. } \\
\text { Stress (Pa) }\end{array}$ & $\begin{array}{c}\text { Max. } \\
\text { Displacement } \\
(\mathbf{m m})\end{array}$ \\
\hline $\begin{array}{c}\text { Straight } \\
\text { Bracket }\end{array}$ & 11864806 & 1,55076 E-07 \\
\hline $\begin{array}{c}\text { Trapez. } \\
\text { Bracket 5o }\end{array}$ & 11872979 & 1,59855 E-07 \\
\hline $\begin{array}{c}\text { Trapez. } \\
\text { Bracket 8 }\end{array}$ & 11884190 & 1,63252 E-07 \\
\hline
\end{tabular}

Based on the above results it may be concluded that the geometry of the archwire in the states of loading torque and compressive force:

1. Has a local effect only in terms of the distribution of forces around the slot. 
2. The distribution of forces in the base of the bracket is independent of the local geometry of the archwire and the slot.

3. The actions or forces transmitted to the tooth are independent of the local geometry of the archwire and the slot.
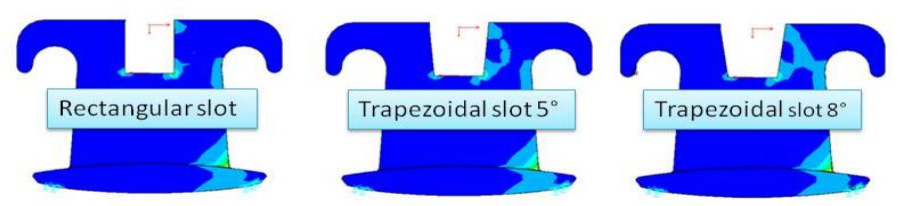

Figure 4. Comparison of the 3 cases.

3.2. Results of the comparative study of archwire friction on different bracket geometries. 1st case

Table 2. Comparative results of the friction study.

\begin{tabular}{|c|c|}
\hline \multicolumn{2}{|c|}{ RESULTS } \\
\hline $\begin{array}{c}\text { Rectangular bracket with } \\
\text { rectangular archwire }\end{array}$ & 4.9504 \\
\hline $\begin{array}{c}\text { Trapezoidal bracket with } \\
\text { trapezoidal archwire }\end{array}$ & 4.9068 \\
\hline $\begin{array}{c}\text { Trapezoidal bracket with } \\
\text { rectangular archwire } \\
\text { supported on slot wall }\end{array}$ & 3.5005 \\
\hline
\end{tabular}

As can be seen from the data included in Table 2, the results of the frictional forces vary considerably depending on the loading case. Although the value of the applied forces is the same in all cases, the geometry of the bodies on which they are applied varies, resulting in a variation in the normal components associated with these external forces.

From these results, it can be concluded that:

Brackets with rectangular slots generate greater frictional forces than those with a trapezoidal geometry. For small angle values of the trapezoidal slot (between $0.1^{\mathrm{o}}-1^{\mathrm{o}}$ ), the influence on the resulting frictional force is very small, as this depends directly on its cosine component.

In the case of rotation of the archwire relative to the bracket, we assume that contact occurs on a single surface. The component of the reaction force, perpendicular to the surface, is clearly smaller than the sum of the normal components applied to both faces. The frictional force associated with the former normal component will therefore also be less than in the case of contact with two surfaces. Comparing the "trapezoidal bracket/trapezoidal archwire" with the "trapezoidal bracket/rectangular archwire on the slot wall", a decrease of $29 \%$ is observed when only one contact surface is involved instead of two.

\subsection{Results of the comparative study of archwire} friction on different bracket geometries - varying the trapezoidal slot angle between $1^{\circ}$ and 10‥2nd case (figs. 5-8)

For all hypotheses of movement studied here, it can be concluded that the frictional force always decreases with increasing angle of the trapezoidal slot.

In term of the different postulated cases, the lowest frictional force is found in the archwire that slides along the slot wall until it is supported on its base, without the possibility of it sliding on the latter.

Due to the large number of variables involved in the movement of the archwire inside the bracket, it cannot be affirmed that the same case of impending movement always occurs. That is, the 4 cases of impending movement are not exclusive and, at any one time, one and/or another may occur.

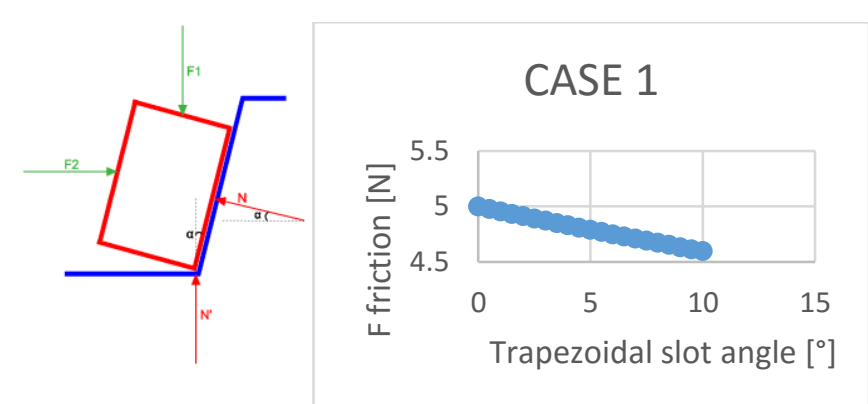

Figure 5. Results of Case 1.
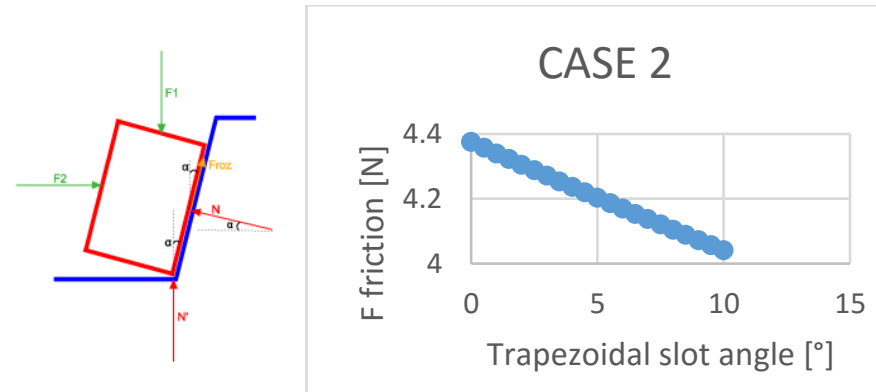

Figure 6. Results of Case 2. 

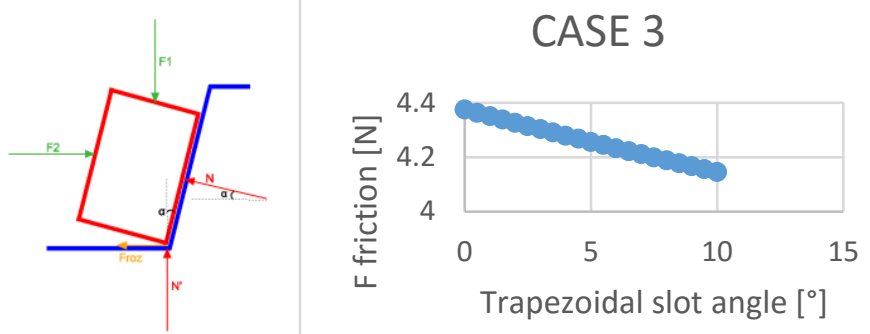

Figure 7. Results of Case 3.
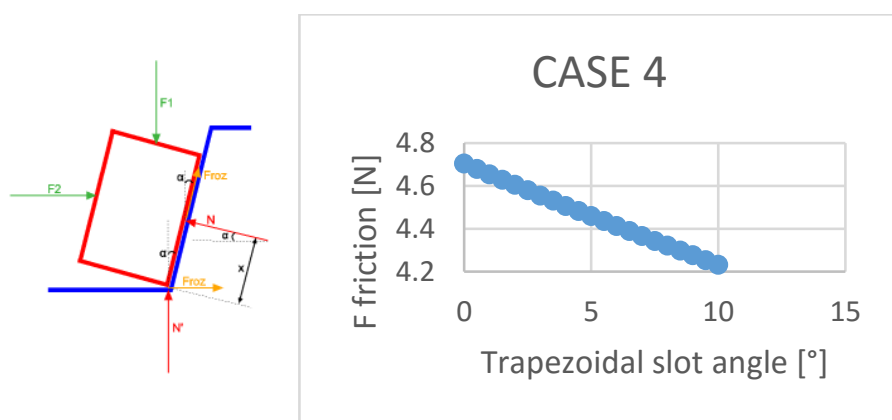

Figure 8. Results of Case 4.

Carrying out a percentage comparison, the most notable decrease in frictional force is produced in the case of the archwire that slides on the slot wall until it is supported on its base, without the possibility of sliding on the latter. This decrease was calculated with respect to the value of the theoretical frictional force $(5 \mathrm{~N})$, which would be the value in the case of a rectangular bracket and rectangular archwire.

If a torque is produced in the archwire, what is most likely to occur (failing a study to verify this) is that the increasing angle would constitute an adverse factor hindering the rotation of the tooth.

If the direction or sign of the assumed forces are varied (once again failing a study to verify this), increasing the angle of the bracket slot could be counterproductive.

To evaluate the putative differential biological effects of the two types of brackets compared here (trapezoidal versus rectangular) enzyme-linked immunosorbent assay, was performed on samples of the gingival crevicular fluid obtained from patients free of periodontal disease (age range 16 to 32 years, 6 females and 6 males) undergoing orthodontic treatment with both kinds of brackets ( $n=6$ for each group). Moreover, samples of gingiva were surgically excised from those patients ( $\mathrm{n}=2$ for each group) from zones exposed to orthodontic forces and processed for Westernblot and immunohistochemistry. A group of 5 subjects with similar ages and of both sexes free of periodontal disease and not undergoing orthodontic treatment were used as controls. This study was approved by the Ethics Committee of Instituto Asturiano de Odontologia (Oviedo, Spain) and informed consent was obtained from each subject. The enzyme-linked immunoassay test was performed using assay kit according to the manufacturer's protocol (R\&D Systems, Minneapolis, MN, USA). The antibodies specific for periostin generated by the entire immunogen were utilized and recombinant human periostin was used to generate a standard curve (see Balli et al. (2015). The studies of Westernblot and double immunofluorescence were performed following Cobo et al. (2015). The antibody used was a rabbit polyclonal antibody against peptide from fasciclin domain 1 of mouse periostin (LS-BL10443, LifeSpan BioSciences, Inc., Seattle, WA, USA). In all patients periostin was detected the values being In the crevicular fluid the amount of periostin were 108,14 \pm $28,15 \mathrm{ng} / \mathrm{ml}$ in control subjects, $122,01 \pm 31,14 \mathrm{ng} / \mathrm{ml}$ in patients undergoing orthodontic treatment with rectangular brackets, and $144,00 \pm 35,14 \mathrm{ng} / \mathrm{ml}$ in patients undergoing orthodontic treatment with trapezoidal brackets. Differences between controls and experimental groups, as well as between patients with both types of brackets, were significant statistically $(\mathrm{p}<0,05$; one way ANOVA). In gingival homogenates Westernblot detected a single protein band with an estimated molecular weight of $94 \mathrm{kDa}$ (Fig. 9a) which is consistent with than expected for the human periostin. Relative periostin levels were increased in both experimental groups (red bars) with respect to the control ones (blue bars) (Fig. 9b). To map the histological distribution of periostin in the human gingiva of the control and experimental patients we used double immunofluorescence coupled with laser-confocal microscopy. In the sections processed for simultaneous demonstration of periostin (green) and vimentin (red) (Figs. 9c-e), periostin immunoreactivity was observed that periostin-immunoreactivity is concentrated at the epithelium-connective tissue junction, presumably associated to ECM proteins of the basal membrane and was never found within the cytoplasm of the basal epithelial cells, and never co-localized with the vimentinpositive fibroblasts, in all the subjects examined. Because the role of periostin in tooth anchorage present results suggest that from a biological point of view the trapezoidal brackets are more effective that the rectangular ones for orthodontic treatments. 


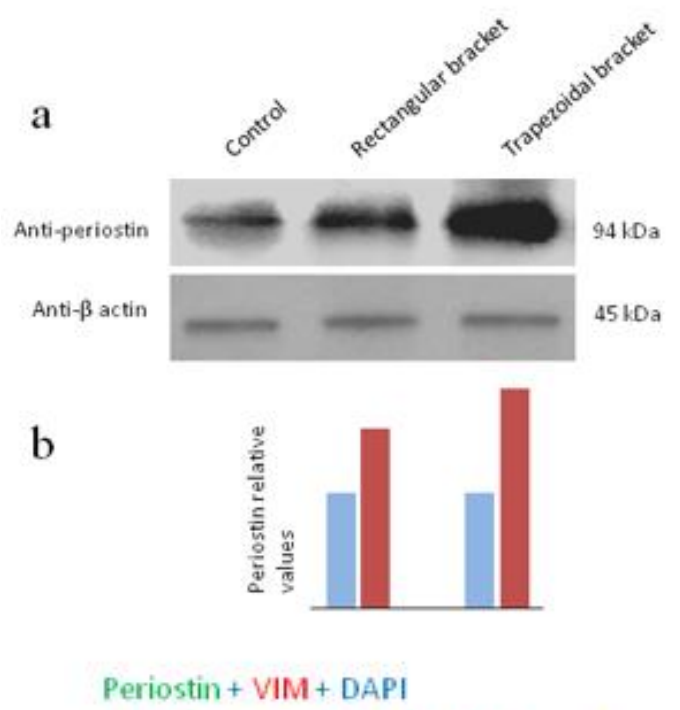

Periostin + VIM + DAPI
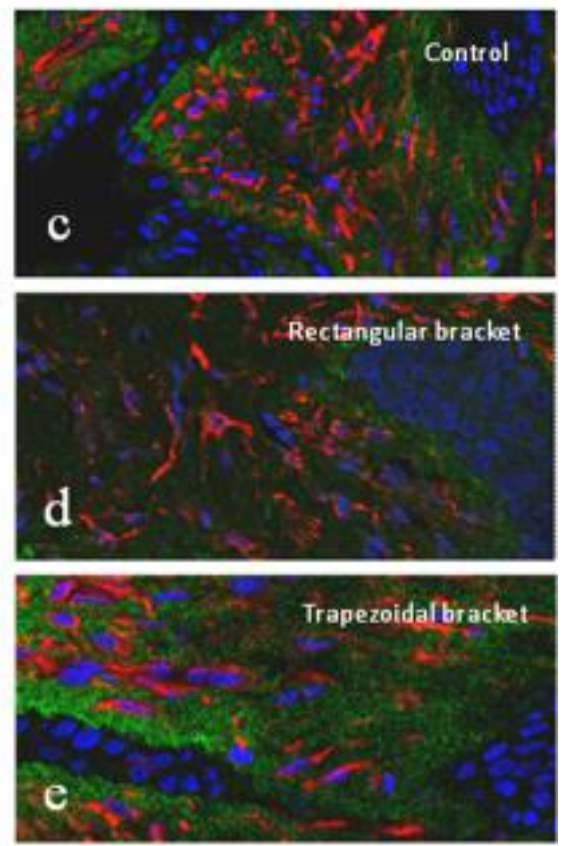

Figure 9. Periostin results. Westernblot (a), relative levels (b) and immunohistochemical localization (a-e) of periostin in the three groups of subjects considered.

\section{Conclusions}

The distribution of forces transmitted to the tooth is more uniform in the trapezoidal than in the rectangular bracket.

Brackets with rectangular slots generate higher frictional forces than those with a trapezoidal geometry. The frictional force always decreases with increasing angle of the trapezoidal slot.

The lowest frictional force is produced when the archwire slides along the slot wall until it is supported on its base, without the possibility of sliding on the latter.
The study of the frictional forces between the bracket and archwire show that a greater bracket angle (regardless of the assumed hypothesis) produces a decrease in the frictional force of advancement in both the rectangular and trapezoidal archwire.

The maximum recommended angle to manufacture brackets is $8^{\circ}$.

All the preceding results lead us to conclude that the best combination is the trapezoidal bracket with a trapezoidal archwire, and therefore trapezoidal brackets should be used preferentially (Figs. 10 to 12).

Furthermore, our research provides new evidence on the impact of periostin in the physiology of tooth movement resulting from the application of orthodontic forces using an archwire bracket device with rectangular and trapezoidal slot walls.

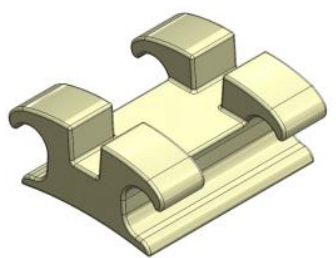

Figure 10. Design of the innovative trapezoidal bracket.

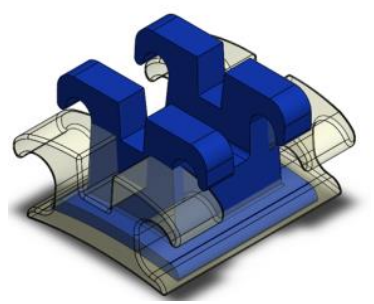

Figure 11. Comparison of the change in dimensions of the trapezoidal versus conventional bracket.

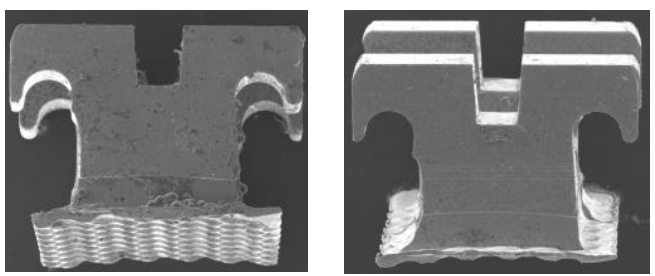

Figure 12. Surface topography of the trapezoidal bracket using a scanning electron microscope.

\section{Acknowledgment}

This research was supported by Instituto Asturiano de Odontología, Principado de Asturias, Spain.

\section{Conflict of Interest}

The authors declare that they have no conflict of interest. 


\section{References}

[1] J. K. Wong, D. L. Romanyk, R. W. Toogood, G. Heo, J. P. Carey and P. W. Major, "The effect of perturbations on resistance to sliding in secondorder moments comparing two different bracket types," J Dent Biomech, vol. 5, pp. 1-8, 2014.

[2] M. R. G. Monteiro, L. E. da Silva, C. N. Elias and O. V. Vilella, "Frictional resistance of self-ligating versus conventional brackets in different bracketarchwire-angle combinations," J Appl Oral Sci., vol. 22, no. 3, pp. 228-34, 2014.

[3] Y. Yanasea, H. Loib, M. Nishiokaa and I. Takahashi, "Effects of sliding velocity on friction. An in vitro study at extremely low sliding velocity approximating orthodontic tooth movement," Angle Orthodontist, vol. 84, no. 3, pp. 451-458, 2014.

[4] R. C. Araujoa, L. M. Bicharab, d. Araujob, A. M. and D. Normando, "Debris and friction of self-ligating and conventional orthodontic brackets after clinical use," Angle Orthodontist, 2014.

[5] J.-Y. Tominaga, H. Ozaki, P.-C. Chiang, M. Sumi, M. Tanaka, Y. Koga, C. Bourauel and N. Yoshida, "Effect of bracket slot and archwire dimensions on anterior tooth movement during space closure in sliding mechanics: A 3-dimensional finite element study," American Journal of Orthodontics and Dentofacial Orthopedics, vol. 146, no. 2, pp. 166174, 2014.

[6] U. Balli, Z. P. Keles, B. Avci, S. Guler, B. O. Cetinkaya and G. C. Keles, "Assessment of periostin levels in serum and gingival crevicular fluid of patients with periodontal disease," J Periodontal Res., 2015.

[7] T. Cobo, A. Obaya, S. Cal, L. Solares, R. Cabo, J. A. Vega and J. Cobo, "Immunohistochemical localization of periostin in human gingiva," Eur J Histochem, 2015. 